\title{
Face-width of Pfaffian Braces and Polyhex Graphs on Surfaces
}

\author{
Dong Ye \\ Department of Mathematical Sciences \\ Middle Tennessee State University \\ Murfreesboro, TN 37132, USA \\ dong. ye@mtsu.edu
}

\author{
Heping Zhang* \\ School of Mathematics and Statistics \\ Lanzhou University \\ Lanzhou, Gansu 730000, China \\ zhanghp@lzu.edu.cn
}

Submitted: July 5, 2013; Accepted: Nov 7, 2014; Published: Nov 20, 2014

Mathematics Subject Classifications: 05C10, 05C70

\begin{abstract}
A graph $G$ with a perfect matching is Pfaffian if it admits an orientation $D$ such that every central cycle $C$ (i.e. $C$ is of even size and $G-V(C)$ has a perfect matching) has an odd number of edges oriented in either direction of the cycle. It is known that the number of perfect matchings of a Pfaffian graph can be computed in polynomial time. In this paper, we show that every embedding of a Pfaffian brace (i.e. 2-extendable bipartite graph) on a surface with a positive genus has face-width at most 3. Further, we study Pfaffian cubic braces and obtain a characterization of Pfaffian polyhex graphs: a polyhex graph is Pfaffian if and only if it is either non-bipartite or isomorphic to the cube, or the Heawood graph, or the Cartesian product $C_{k} \times K_{2}$ for even integers $k \geqslant 6$.
\end{abstract}

Keywords: Pfaffian orientation; perfect matching; polyhex graphs; embedding

\section{Introduction}

Let $G$ be a graph with vertex set $V(G)$ and edge set $E(G)$. A perfect matching of $G$ is a set $M$ of independent edges such that every vertex of $G$ is incident with exactly one edge in $M$. The number of perfect matchings of a graph has applications in statistic physics and quantum chemistry and usually is difficult to compute (cf. [6, 13]). A subgraph $H$ of a graph $G$ is central if $G-V(H)$ has a perfect matching. If $G$ has a perfect matching, a central cycle $C$ must be of even size. In other words, $G$ has a perfect matching $M$ such that $C$ is $M$-alternating (i.e. the edges of $C$ alternate between $M$ and $E(G) \backslash M$ ). Let

*Research supported by NSF of China Grant (No. 11371180). 
$D$ be an orientation of $G$. An even cycle $C$ is oddly oriented if $C$ has an odd number of edges directed in either direction of the cycle. An orientation of $G$ is Pfaffian if every central cycle of $G$ is oddly oriented. A graph $G$ is Pfaffian if it has a Pfaffian orientation. It is known that the number of perfect matchings of a Pfaffian graph can be obtained by computing the determinant of the skew adjacency matrix of its Pfaffian orientation $D$ (cf. [13]).

The Pfaffian orientation was first introduced by Kasteleyn [6] for solving 2-dimensional Ising problem, and Kasteleyn [6] showed that every planar graph admits a Pfaffian orientation. The problem of characterizing Pfaffian bipartite graphs is related to many interesting problems, such as the Pólya permanent problem, the sign-nonsingular matrix problem, etc, and gets a lot of attention $([11,14,21])$. Little [11] proved that a bipartite graph is Pfaffian if and only if it does not contain an even-subdivision of $K_{3,3}$ as a central subgraph. However, Little's characterization does not provide a polynomial time algorithm to determine Pfaffian bipartite graphs.

A connected graph $G$ is $k$-extendable $(|V(G)| \geqslant 2 k+2)$ if $G$ has $k$ independent edges and any $k$ independent edges are contained in a perfect matching of $G$. A 2-extendable bipartite graph is called a brace. A $k$-extendable graph is $(k-1)$-extendable and $(k+1)$ connected [19, 13]. So a brace is 3-connected. A graph $G$ is bicritical if, for any two vertices $u$ and $v$ of $G$, the subgraph $G-\{u, v\}$, obtained from $G$ by deleting $u$ and $v$, has a perfect matching. A bicritical graph with more than two vertices is 1-extendable and nonbipartite. A brick is a 3-connected bicritical graph. By Lovász's tight-cut decomposition $[12,13]$, every 1-extendable graph can be reduced to a list of braces and bricks. Vazirani and Yannakakis [27] showed that a graph $G$ is Pfaffian if and only if all braces and bricks generated from tight-cut decomposition are Pfaffian. Robertson, Seymour and Thomas [21], and independently McCuaig [14] obtained an elegant characterization of Pfaffian braces, which leads to a polynomial time algorithm to determine whether a given braces is Pfaffian or not. The characterization problem remains open for non-bipartite graphs and graphs on surfaces with positive genera. Readers may refer to the survey of Thomas [24] on the Pfaffian orientations of graphs.

Let $G$ be a graph and $\Pi$ an embedding of $G$ on a surface $\Sigma$. A closed simple curve $\ell$ in $\Sigma$ is contractible if $\Sigma-\ell$ has precisely two components and one of them is homeomorphic to an open disk. The face-width (or representativity) of $\Pi$ is the maximum integer $k$ such that every non-contractible simple closed curve in $\Sigma$ intersects the graph at least $k$ points (see [15]), denoted by $\mathrm{fw}(G, \Pi)$. For convenience, assume that a plane graph has facewidth infinity. Robertson and Vitray [22], and independently Thomassen [25], showed that a planar graph embedded on a surface $\Sigma$ with a positive genus has the face-width at most 2 .

A polyhex graph is a cubic graph cellularly embedded on a surface such that every face is bounded by a hexagon. By Euler's formula, the surface could be only the torus and the Klein bottle. Polyhex graphs have been studied as surface tilings [16, 26], and are also considered as possible generalizations of fullerenes in chemistry and material science $[2,8,9]$. A detailed classification of polyhex graphs was given by Thomassen [26]. A bipartite polyhex graph is a cubic brace [29]. In [5], P.E. John tried using the Pfaffian 
method to enumerate the perfect matchings of polyhex graphs on the torus. However, not all polyhex graphs on torus are Pfaffian.

In this paper, we study Pfaffian graphs embedded on surfaces with positive genera. The paper is organized as follows. In Section 2, we show that every embedding of a Pfaffian brace embedded on a surface with a positive genus has face-width at most 3 . It is natural to ask whether a Pfaffian brick has bounded face-width. In the end of this paper, we give a negative answer to this question via non-bipartite polyhex graphs. In Section 3, we obtain some structure properties of Pfaffian cubic braces, which are applied to characterize Pfaffian polyhex graphs. In Section 4, the construction of polyhex graphs is presented and polyhex graphs with small face-width have been determined. In Section 5, we completely characterize Pfaffian polyhex graphs. We show that a polyhex graph is Pfaffian if and only if it is either non-bipartite or isomorphic to the cube, or the Heawood graph, or the Cartesian product $C_{k} \times K_{2}$ for even integer $k \geqslant 6$.

\section{Face-width of Pfaffian braces on surfaces}

Let $G$ be a graph cellularly embedded on a surface $\Sigma$ (each face is homeomorphic to an open disk in the plane). For a face $f$ of $G$, the boundary of $f$ is often represented by a closed walk of $G$, denoted by $\partial f$, also called a facial walk. An embedding $\Pi$ is a strong embedding if every facial walk is a cycle. The face-width $\mathrm{fw}(G, \Pi)$ of an embedding $\Pi$ of $G$ on a surface $\Sigma$ is the smallest number $k$ such that there exist $k$ faces whose union contains a non-contractible curve of $\Sigma$. Undefined notations and concepts are referred to [15].

The following result presents an important property of a planar graph embedded on a surface $\Sigma$ with genus $g(\Sigma)>0$.

Theorem 1 ([22, 25]). Let $G$ be a planar 3-connected graph. Then every embedding of $G$ on a surface $\Sigma$ with genus $g(\Sigma)>0$ has face-width at most 2 .

Kasteleyn [7] showed that every planar graph is Pfaffian. But the above result does not hold for all Pfaffian graphs. For example, the Heawood graph, a Pfaffian brace (a Pfaffian orientation is shown in Figure 1), admits an embedding on the torus with face-width 3 (see Lemma 2.3 and Lemma 4.7).

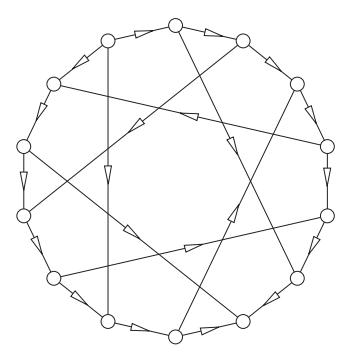

Figure 1: The Heawood graph with a Pfaffian orientation. 
Lemma 2 (Prop. 5.5.12 on Page 150 in [15]). Let $G$ be a graph and $\Pi$ be an embedding of $G$ on a surface $\Sigma$. Then the following conditions are equivalent:

(1) $\mathrm{fw}(G) \geqslant 3$ and $G$ is 3-connected;

(2) All facial walks of $\Pi$ are cycles and any two of them are either disjoint or their intersection is just a vertex or an edge.

Lemma 3. Every embedding of the Heawood graph on a surface $\Sigma$ has face-width at most 3.

Proof. Let $\Pi$ be an embedding of the Heawood graph $G$ on a surface $\Sigma$. Suppose to the contrary that $\operatorname{fw}(G, \Pi)=k \geqslant 4$. Then there exist $k$ faces $f_{0}, f_{1}, f_{2}, \ldots, f_{k-1}$ such that their union contains a non-contractible closed curve $\ell$ of $\Sigma$ such that $k$ is minimum. Since $G$ is 3 -connected, every $\partial f_{i}$ is a cycle by Lemma 2 . By the minimality of $k$ and Lemma 2 , it follows that $f_{i}$ and $f_{j}$ share an edge if and only if $|j-i| \equiv 1(\bmod k)$. In other words, $\left|V\left(\partial f_{i} \cap \partial f_{j}\right)\right| \geqslant 2$ if $|i-j| \equiv 1(\bmod k)$, and $\left|V\left(\partial f_{i} \cap \partial f_{j}\right)\right|=0$ otherwise. Note that $\left|V\left(\partial f_{i}\right)\right| \geqslant 6$ since $G$ has the girth six. So

$$
14=|V(G)| \geqslant\left|V\left(\bigcup_{i=0}^{k-1} \partial f_{i}\right)\right|=\sum_{i=0}^{k-1} V\left(\partial f_{i}\right)-\sum_{i=0}^{k-1}\left|V\left(\partial f_{i} \cap \partial f_{i+1}\right)\right| \geqslant 6 k-2 k=4 k \geqslant 16,
$$

a contradiction. This completes the proof.

The following result shows that the above result holds for all Pfaffian braces.

Theorem 4. Let $G$ be a Pfaffian brace. Then every embedding of $G$ on a surface $\Sigma$ with $g(\Sigma)>0$ has face-width at most 3 .

In order to prove Theorem 4, we need the characterization of Pfaffian braces obtained by Robertson, Thomas and Seymour [21], and independently by McCuaig [14]. Let $G_{0}$ be a graph and $C$ a central 4-cycle of $G_{0}$; that means, $G_{0}-V(C)$ has a perfect matching. Let $G_{1}, G_{2}$ and $G_{3}$ be three subgraphs of $G_{0}$ such that $G_{1} \cup G_{2} \cup G_{3}=G_{0}$, and for distinct $i, j \in\{1,2,3\}, G_{i} \cap G_{j}=C$ and $V\left(G_{i}\right)-V(C) \neq \emptyset$. A graph $G$ is a tri-sum of $G_{1}, G_{2}$ and $G_{3}$ if it is obtained from $G_{0}$ by deleting some edges (possibly none) of $C$. For example, Figure 2 shows a cubic brace generated from three copies of the cube by the tri-sum operation along the cycle $C$ illustrated by dash lines (all edges of $C$ are deleted in the operation). By a result of McCuaig (Lemma 19 on page 36 in [14]), every tri-sum of three braces is a new brace. The following is a variant of the statement of the characterization of Pfaffian braces obtained in [14, 21].

Theorem 5 ([21, 14], Theorem 4.2 in [24]). A bipartite graph is a Pfaffian brace if and only if it is isomorphic to the Heawood graph, or it can be obtained from planar braces by repeated application of the tri-sum operation.

Let $G$ be a graph, and $G_{1}$ and $G_{2}$ be two disjoint subgraphs of $G$. Denote the set of edges joining vertices of $G_{1}$ and vertices of $G_{2}$ by $E\left(G_{1}, G_{2}\right)$. For a vertex $w$ of $G$, let $N(w)$ be the set of all neighbors of $w$ in $G$. The following technical lemma will be used to prove Theorem 4. 


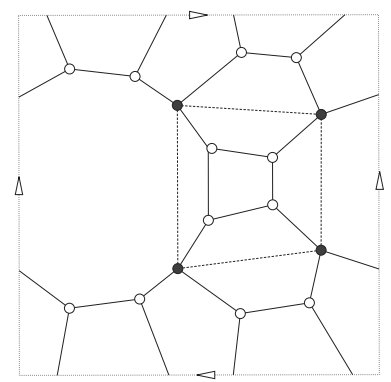

Figure 2: A cubic brace on the Klein bottle generated from three copies of the cube by the tri-sum operation (edges of the cycle $C$ are illustrated by dash lines).

Lemma 6. Let $G$ be a 3-connected graph and $W$ be a vertex-cut of size 4. If $G-W$ can be decomposed into three disjoint graphs $G_{1}, G_{2}$ and $G_{3}$ such that for distinct $i, j \in\{1,2,3\}$ and $w \in W, E\left(G_{i}, G_{j}\right)=\emptyset$ and $N(w) \cap V\left(G_{i}\right) \neq \emptyset$, then every embedding of $G$ on a surface with a positive genus has face-width at most 3 .

Proof. Let $\Pi$ be an embedding of $G$ on a surface $\Sigma$ with $g(\Sigma)>0$. Suppose on the contrary that $\mathrm{fw}(G, \Pi)>3$.

Let $W=\left\{v_{1}, v_{2}, v_{3}, v_{4}\right\}$ and $E(W)$ be the set of all edges with two end-vertices in $W$. For any $v_{i} \in W$, let $v_{i} v_{i, 0}, \ldots, v_{i} v_{i, k_{i}}$ be the edges incident with $v_{i}$ in clockwise direction in some small neighborhood of $v_{i}$ homeomorphic to an open disc. Note that $N\left(v_{i}\right) \cap V\left(G_{\alpha}\right) \neq \emptyset$ for every $\alpha \in\{1,2,3\}$. Let $f_{i, \alpha}$ be a face containing $v_{i} v_{i, \alpha_{i}}$ and $v_{i} v_{i, \alpha_{i}+1}$ such that $v_{i, \alpha_{i}} \in V\left(G_{\alpha}\right)$ and $v_{i, \alpha_{i}+1} \notin V\left(G_{\alpha}\right)$. For an edge $v_{i} v_{i, k} \in E(W)$, let $f$ be the face containing $v_{i} v_{i, k}$ and $v_{i} v_{i, k+1}$. By the definition of faces $f_{i, \alpha}$ and the ordering of edges incident with $v_{i}, f \neq f_{i, \alpha}$ for any $\alpha \in\{1,2,3\}$ because $v_{i, k} \notin G-W$. So the intersection of two distinct faces $f_{i, \alpha}$ and $f_{i, \beta}$ does not contain edges of $E(W)$ incident with $v_{i}$.

Claim 1. The intersection of two distinct faces $f_{i, \alpha}$ and $f_{i, \beta}$ does not contain another vertex from $W$ different from $v_{i}$.

Proof of Claim 1. Note that $G$ is 3-connected and $\operatorname{fw}(G, \Pi)>3$. By Lemma 2, the intersection of $f_{i, \alpha}$ and $f_{i, \beta}$ is either $v_{i}$ or an edge $v_{i} v_{i, k}$. Since $f_{i, \alpha} \cap f_{i, \beta}$ does not contain an edge from $E(W)$ incident with $v_{i}$, it follows that $v_{i, k} \notin W$. So Claim 1 holds.

Claim 2. Every $f_{i, \alpha}$ contains precisely two vertices of $W$.

Proof of Claim 2. First, we show that every $f_{i, \alpha}$ contains at least two vertices of $W$. By Lemma 2, each $f_{i, \alpha}$ is bounded by a cycle. Note that $v_{i, \alpha_{i}} \in V\left(G_{\alpha}\right), v_{i, \alpha_{i}+1} \notin V\left(G_{\alpha}\right)$. If $v_{i, \alpha_{i}+1} \in W$, then $f_{i, \alpha}$ contains two vertices from $W$. So suppose that $v_{i, \alpha_{i}+1} \notin W$. Hence, $\partial f_{i, \alpha}-W$ consists of at least two components: one contains $v_{i, \alpha_{i}}$ and the other contains $v_{i, \alpha_{i}+1}$. These two components are joined by vertices from $W$ because $E\left(G_{\alpha}, G_{\beta}\right)=\emptyset$ for distinct $\alpha, \beta \in\{1,2,3\}$. So $f_{i, \alpha}$ contains at least two vertices from $W$.

Since $N\left(v_{i}\right) \cap V\left(G_{\alpha}\right) \neq \emptyset$, there are at least three distinct faces $f_{i, \alpha}(\alpha \in\{1,2,3\})$ incident with $v_{i}$. If one of them contains three vertices from $W$, then there is a pair of 
vertices which are contained by two distinct faces $f_{i, \alpha}$ and $f_{i, \beta}$, contradicting Claim 1 . So Claim 2 follows.

By Claim 1 and Claim 2, we further have the following conclusion:

Any two vertices of $W$ are contained by some face $f_{i, \alpha}$, and every face $f_{i, \alpha}$ contains precisely a pair vertices of $W$.

Without loss of generality, assume that $f_{1,1}$ contains $v_{1}$ and $v_{2}$, and $f_{1,2}$ contains $v_{1}$ and $v_{4}$, and $f_{1,3}$ contains $v_{1}$ and $v_{3}$ (relabeling $G_{\alpha}$ for $\alpha=1,2,3$ if necessary). Then, assume that $v_{2}$ and $v_{4}$ are contained by $f_{2, \alpha}, v_{2}$ and $v_{3}$ are contained by $f_{2, \beta}$ with $\beta \neq \alpha, v_{3}$ and $v_{4}$ are contained by $f_{3, \mu}$ for some $\mu \in\{1,2,3\}$. (For example, see Figure 3 (left). The shadow parts illustrate the regions of $\Sigma$ containing vertices and edges from only $G_{\alpha}-W$ for some $\alpha \in\{1,2,3\}$, or one edge from $E(W)$.) Then each of $f_{1,1} \cup f_{2, \beta} \cup f_{1,3}, f_{1,1} \cup f_{2, \alpha} \cup f_{1,2}$, $f_{1,2} \cup f_{3, \mu} \cup f_{1,3}$ and $f_{2, \alpha} \cup f_{2, \beta} \cup f_{3, \mu}$ contains a closed curve which intersects $G$ at three vertices of $W$. Denote these closed curves by $\ell_{1}, \ell_{2}, \ell_{3}$ and $\ell_{4}$.
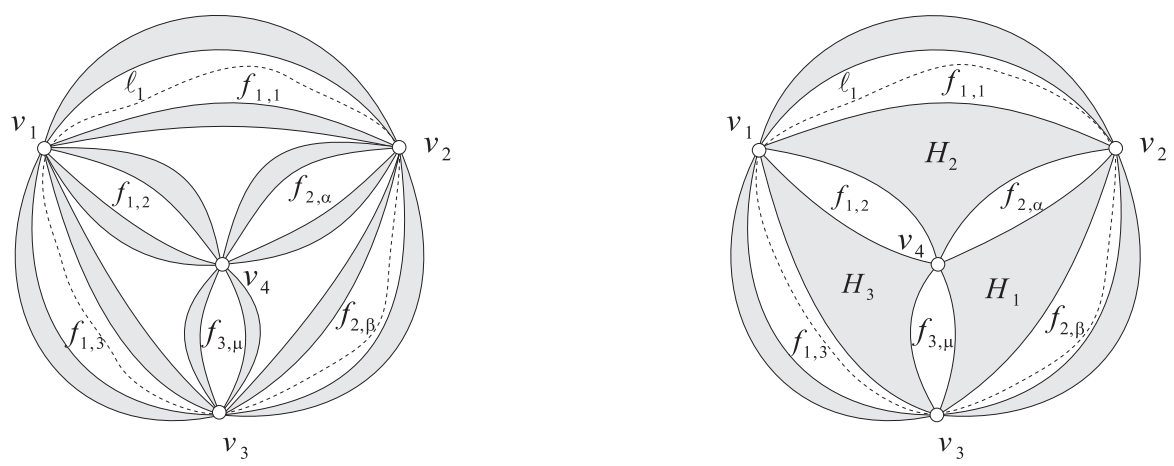

Figure 3: Illustration for the proof of Theorem 4.

Claim 3. At least one of the closed curves $\ell_{i}(i=1, \ldots, 4)$ is non-contractible.

Proof of Claim 3. If not, suppose that all $\ell_{i}(i=1, \ldots, 4)$ are contractible. Then each $\ell_{i}$ separates $\Sigma$ into two regions, and at least one of them is homeomorphic to an open disc, denoted by $D_{i}$.

First, suppose that $v_{4}$ lies on $D_{1}$. Let $R_{1} \subseteq D_{1}$ be the region bounded by faces $f_{1,1}$, $f_{1,2}$ and $f_{2, \alpha}$.

By the definition of $f_{1,2}, R_{1}$ contains a vertex from $G_{2}$. Let $H_{2}$ be the subgraph of $G$ (not including $v_{1}, v_{2}$ and $v_{4}$ ) embedded in $R_{1}$. By Claim 1 , every face of the embedding of the subgraph $H_{2}$ together with $v_{1}, v_{2}$ and $v_{4}$ inherited from the embdding $\Pi$ of $G$ in the region $R_{1}$ contains at most one vertex from $v_{1}, v_{2}$ and $v_{4}$. So $H_{2} \subseteq G_{2}$. Similarly, the subgraph $H_{3}$ (not including $v_{1}, v_{3}$ and $v_{4}$ ) in the region $R_{2} \subseteq D_{1}$ bounded by faces $f_{1,2}$, $f_{1,3}$ and $f_{3, \mu}$ is a subgraph of $G_{3}$. Further, the subgraph $H_{1}$ (not including $v_{2}, v_{3}$ and $v_{4}$ ) in the region $R_{3} \subseteq D_{1}$ bounded by faces $f_{2, \alpha}, f_{2, \beta}$ and $f_{3, \mu}$ is a subgraph of $G_{1}$ (see Figure 3 (right)). 
Let $G^{\prime}$ be the subgraph (including $v_{1}, v_{2}$ and $\left.v_{3}\right)$ in $\Sigma-D_{1}$. If $V\left(G^{\prime}\right)=\left\{v_{1}, v_{2}, v_{3}\right\}$, then $G$ is embedded in an open disc of $\Sigma$. Hence $\mathrm{fw}(G, \Pi)=0$ because $g(\Sigma)>0$.

So suppose that $G^{\prime}$ contains at least one vertex $w$ different from $v_{1}, v_{2}$ and $v_{3}$. Let $H_{\alpha}^{\prime}=G^{\prime} \cap G_{\alpha}$. Then all vertices $v_{1}, v_{2}$ and $v_{3}$ have a neighbor in $H_{\alpha}^{\prime}$ since $G$ is 3 connected. If two of $H_{1}^{\prime}, H_{2}^{\prime}$ and $H_{3}^{\prime}$ are not empty, then there exists a face $f_{1, \gamma}$ for some $\gamma \in\{1,2,3\}$ which contains a vertex $v_{1, \gamma_{i}} \in H_{\gamma}^{\prime}$ and a vertex $v_{1, \gamma_{i}+1} \notin H_{\gamma}^{\prime}$. By Claim 1, $f_{1, \gamma}$ contains two vertices from $W$. Then there are two distinct faces, one $f_{1, \gamma}$ and another from $f_{1,1}, f_{1,2}, f_{1,3}$ contains the same pair of vertices from $W$, a contradiction. So only one of $H_{1}^{\prime}, H_{2}^{\prime}$ and $H_{3}^{\prime}$ is not empty. Without loss of generality, assume $H_{1}^{\prime} \neq \emptyset$ and hence $G^{\prime}-W \subset G_{1}$.

Then $G_{2}=H_{2}$. Note that $N\left(v_{3}\right) \cap V\left(G_{2}\right)=\emptyset$, contradicting that $N\left(v_{i}\right) \cap V\left(G_{\alpha}\right) \neq \emptyset$ for any $v_{i} \in W$ and any $\alpha \in\{1,2,3\}$. The contradiction implies that $v_{4}$ does not lie on $D_{1}$.

By symmetry, the region $D_{i}(i \in\{1,2,3,4\})$ does not contain the vertex of $W$ which is not on $\ell_{i}$. Then $\Sigma$ must be the sphere since it is formed by pasting the four disc $D_{i}$ along the four closed curves $\ell_{i}$, contradicting $g(\Sigma)>0$. This completes the proof of Claim 3 .

Claim 3 implies that $\mathrm{fw}(G, \Pi) \leqslant 3$, which contradicts the assumption that $\mathrm{fw}(G, \Pi)>$ 3. The contradiction completes the proof.

A minimal vertex-cut $W$ of a connected graph $G$ is a tri-cut if $G-W$ has at least three components. For any vertex $w$ of a tri-cut $W$ and any component $G_{i}$ of $G-W$, $N(w) \cap V\left(G_{i}\right) \neq \emptyset$. As a direct corollary of Lemma 6, we have the following result.

Theorem 7. Let $G$ be a 3-connected graph with a tri-cut of size 4. Then every embedding of $G$ on a surface with a positive genus has face-width at most 3.

Now we are ready to prove Theorem 4 .

Proof of Theorem 4: Let $G$ be a Pfaffian brace. If $G$ is the Heawood graph or a planar brace, the result follows from Lemma 3 and Theorem 1 . Otherwise, by Theorem $5, G$ is generated from planar Pfaffian braces by applying the tri-sum operations. Assume that $G$ is generated from Pfaffian braces $G_{1}, G_{2}$ and $G_{3}$ by the tri-sum operation along a central cycle $C=v_{1} v_{2} v_{3} v_{4} v_{1}$. Since each $G_{i}$ is 3-connected and bipartite, $N\left(v_{j}\right) \cap\left(V\left(G_{i}\right) \backslash V(C)\right) \neq$ $\emptyset$ for every $v_{j} \in V(C)$. Note that $G$ itself is a brace and hence 3-connected. So $G$ and $W=V(C)$ satisfy the conditions of Lemma 6 . Hence every embedding of $G$ on a surface with a positive genus has face-width at most 3 by Lemma 6 .

\section{Pfaffian cubic braces}

In order to characterize Pfaffian polyhex graphs, we need more structure properties of Pfaffian cubic braces. The following result is a construction for Pfaffian cubic braces which follows from Theorem 5 . 
Theorem 8. A cubic bipartite graph is a Pfaffian brace if and only if it is isomorphic to the Heawood graph, or it can be obtained from planar cubic braces by repeated application of the tri-sum operation.

Proof. If $G$ is a non-planar Pfaffian cubic brace different from the Heawood graph, then by Theorem 5, $G$ is generated from planar braces by tri-sum operations. We may assume that $G$ is generated from three Pfaffian braces $G_{1}, G_{2}$ and $G_{3}$ along a 4-cycle $C$ by the tri-sum operation. Since each $G_{i}$ is 3 -connected and $G$ is cubic, each vertex of $C$ has precisely one neighbor in each $G_{i}-V(C)$. So every $G_{i}$ is cubic. The other direction follows directly from Theorem 5 .

A set $S$ of edges of a connected graph $G$ is a cyclic edge-cut if $G-S$ has two components, each of which contains a cycle. A connected graph $G$ is cyclically $k$-edge-connected if every cyclic edge-cut of $G$ has at least $k$ edges. The cyclic edge-connectivity of $G$ is the maximum integer $k$ such that $G$ is cyclically $k$-edge-connected, denoted by $c \lambda(G)$.

Theorem 9 ([3, 4]). Let $G$ be a cubic bipartite graph. Then $G$ is a brace if and only if it is cyclically 4-edge-connected.

Theorem 10. Let $G$ be a Pfaffian cubic brace different from the Heawood graph. Then $c \lambda(G)=4$. Furthermore, for any 4-cycle $C$ of $G, E(C, G-V(C))$ is a cyclic 4-edge-cut.

Proof. First we prove the following claim:

Claim: If $G$ has a 4-cycle $C$, then $E^{\prime}:=E(C, G-V(C))$ is a cyclic 4-edge-cut.

Proof of Claim: It is obvious that $\left|E^{\prime}\right|=4$. Note that, the only cubic bipartite graph with 6 vertices is $K_{3,3}$, which is not Pfaffian. So $G$ has at least $n \geqslant 8$ vertices. Since $G$ is 3-connected, $G-V(C)$ is connected. It is easily seen that $G-V(C)$ has $n-4$ vertices and $3 n / 2-8$ edges. Note that $3 n / 2-8 \geqslant n-4$ because $n \geqslant 8$. So $G-V(C)$ has a cycle. Hence $E^{\prime}$ is a cyclic 4-edge-cut and the claim holds.

If $G$ is planar, by Euler's formula, $G$ must contain a 4 -cycle since $G$ is bipartite. The claim implies that $G$ contains a cyclic 4-edge cut. If $G$ is non-planar, then $G$ is generated from Pfaffian cubic braces $G_{1}, G_{2}$, and $G_{3}$ by the tri-sum along a 4 -cycle $C$ by Theorem 8 . Since $G$ is not the Heawood graph, the claim implies that each $E\left(C, G_{i}-V(C)\right)$ is a cyclic 4-edge-cut of $G_{i}$, which is also a cyclic 4-edge-cut of $G$ since each $G_{i}-V(C)(i \in\{1,2,3\})$ contains a cycle. So $c \lambda(G) \leqslant 4$. Hence the theorem follows from Theorem 9 .

Let $G$ be a 3-connected graph with a tri-cut $W$ of size 4 . Then $W$ is called an ideal tri-cut if $W$ is an independent set of $G$ and $G-W$ has exactly three components $G_{1}^{\prime}, G_{2}^{\prime}$ and $G_{3}^{\prime}$ such that each $G_{i}^{\prime}$ has at least four vertices, and each $E\left(W, G_{i}^{\prime}\right)$ is a matching covering all vertices of $W$.

Proposition 11. Let $G$ be a Pfaffian cubic brace. If $G$ is neither planar nor the Heawood graph, then $G$ has an ideal tri-cut $W$. 
Proof. By Theorem 8, $G$ is generated from three Pfaffian cubic braces $G_{1}, G_{2}$ and $G_{3}$ by a tri-sum operation along a 4-cycle $C$. Let $W:=V(C)$. Then $W$ is an independent set of $G$. Since every $G_{i}$ is 3-connected and Pfaffian, $G_{i}-V(C)$ is connected and has at least four vertices. Since each vertex of $W$ has a neighbor in every $G_{i}-W$, it follows that $W$ is a minimal vertex-cut of $G$. It remains to show that each $E\left(W, G_{i}-W\right)$ is a matching covering all vertices of $W$. This holds because $E\left(W, G_{i}-W\right)$ is a minimum cyclic edge-cut of $G_{i}$ by Theorem 10 .

\section{Construction of polyhex graphs}

A polyhex graph is a cubic graph embedded on a surface such that every face is a hexagon, a cycle of length 6 . The embedding of a polyhex graph is a strong embedding. By Euler's formula, the surfaces can be only the torus and the Klein bottle [2].

Let $G_{1}$ and $G_{2}$ be two polyhex graphs isomorphic to each other. An isomorphism $\sigma$ from $G_{1}$ to $G_{2}$ is hexagon-preserving if $h$ is a hexagon of $G_{1}$ if and only if $\sigma(h)$ is a hexagon of $G_{2}$. An isomorphism from a graph $G$ to itself is called an automorphism. A graph $G$ is vertex-transitive if, for any two vertices $v_{1}, v_{2} \in V(G)$, there exists an automorphism $\sigma$ such that $\sigma\left(v_{1}\right)=v_{2}$. A polyhex graph $G$ is hexagon-transitive if for any two hexagons $h_{1}$ and $h_{2}$, there exists a hexagon-preserving automorphism $\sigma$ such that $\sigma\left(h_{1}\right)=h_{2}$.

Let $R$ be a rectangle of a 2-dimensional Euclidean plane $\mathbb{R}^{2}$ such that $R=\{(x, y)$ : $0 \leqslant x \leqslant k, 0 \leqslant y \leqslant q\}$, where $k$ and $q$ are positive integers. Let $v_{i, j}$ be a vertex corresponding to the point $(i, j)$ where $i$ and $j$ are non-negative integers. A rectangular hexagon lattice $L(k, q)$ is a graph on $R$ consisting of all vertices $v_{i, j}$ in $R$ and edges in $\left\{v_{i, j} v_{i+1, j} \mid 0 \leqslant j \leqslant q, 0 \leqslant i \leqslant k-1\right\} \cup\left\{v_{i, j} v_{i, j+1} \mid 0 \leqslant i \leqslant k, 0 \leqslant j \leqslant q-1\right.$ and $i \equiv j$ $\bmod 2\}$. For example, $L(6,4)$ is shown in Figure 4.

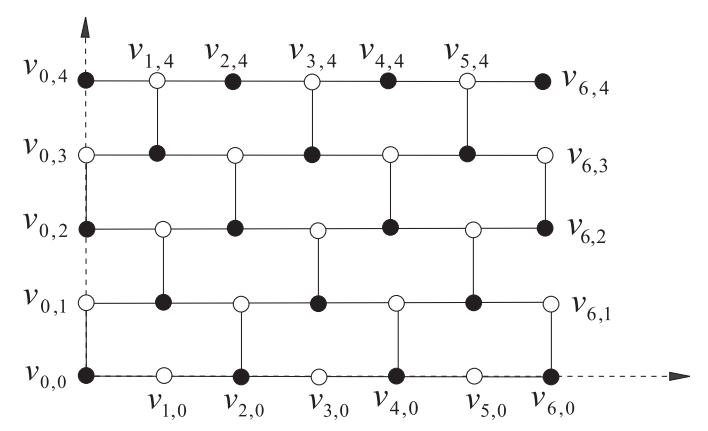

Figure 4: A rectangular hexagon lattice $L(6,4)$.

The vertices $v_{i, j}$ of $L(k, q)$ are colored in black if $i+j \equiv 0(\bmod 2)$ and white if $i+j \equiv 1(\bmod 2)($ see Figure 4$)$. For even $k$, a polyhex tube $L^{\prime}(k, q)$ is obtained from $L(k, q)$ by identifying the vertex $v_{0, j}$ with the vertex $v_{k, j}$ for every $j \in\{0,1, \ldots, q\}$. So both $L(k, q)$ and $L^{\prime}(k, q)$ are bipartite graphs. The cycle $v_{0, i} v_{1, i} \cdots v_{k, i}$ is called the $i$-th 
layer, denoted by $L_{i}$. Let $h_{i, j}$ denote the hexagon with the center at $\left(2 i+\alpha_{j}, j+\frac{1}{2}\right)$ where

$$
\alpha_{j}:=\left\{\begin{array}{lll}
0, & \text { if } j \equiv 1 \quad(\bmod 2) \\
1, & \text { if } j \equiv 0 & (\bmod 2) .
\end{array}\right.
$$

Equivalently,

$$
h_{i, j}:=\left\{\begin{array}{lll}
v_{2 i-1, j} v_{2 i, j} v_{2 i+1, j} v_{2 i+1, j+1} v_{2 i, j+1} v_{2 i-1, j+1} v_{2 i-1, j}, & \text { if } j \equiv 1 & (\bmod 2) \\
v_{2 i, j} v_{2 i+1, j} v_{2 i+2, j} v_{2 i+2, j+1} v_{2 i+1, j+1} v_{2 i, j+1} v_{2 i, j}, & \text { if } j \equiv 0 & (\bmod 2) .
\end{array}\right.
$$

Altschuler [1] showed that polyhex graphs on torus, denoted by $T(k, q, t)$, can be constructed in the following way: From $L^{\prime}(k, q)$ ( $k$ even) identify the vertices $v_{i, 0}$ and $v_{i+q+2 t, q}$ where $0 \leqslant t \leqslant k / 2-1$ and the first subscript is always modulo $k$; that is, $v_{i, 0}$ is connected to $v_{i+q+2 t, q-1}$ by an edge for each odd $i$. For example, see Figure 5.
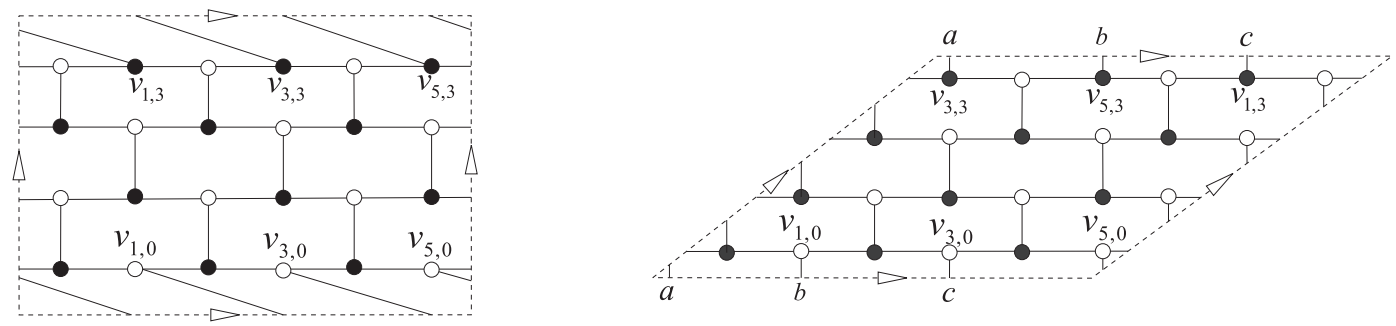

Figure 5: Representations for the polyhex graph $T(6,4,0)$ on the torus.

Since a polyhex graph on the torus is a strong embedding, we have the following result.

Theorem 12. A polyhex graph on the torus is isomorphic to $T(k, q, t)$ for $k \equiv 0(\bmod 2)$ and $(k, q, t) \notin\left\{(2, q, t),(4,1, t),(k, 1,0),(k, 1, k / 2-1) \mid k, q \in \mathbb{Z}^{+}, 0 \leqslant t \leqslant k / 2-1\right\}$.

Thomassen [26] classified polyhex graphs into seven types: two on the torus, five on the Klein bottle. According to Thomassen's constructions, Li et al. [10] reclassified the polyhex graphs on the Klein bottle into the following two types:

- Bipartite polyhex $K_{e}(k, q)\left(q \geqslant 2, k \geqslant 4\right.$ is even): From $L^{\prime}(k, q)$, identify $v_{i, 0}$ with $v_{k-i, q}$ if $q$ is even, and $v_{i, 0}$ with $v_{k-i-1, q}$ if $q$ is odd.

- Non-bipartite polyhex $K_{o}(k, q)(q \geqslant 2$ is even, and $k \geqslant 3)$ : From $L(k, q)$ first identify $v_{i, 0}$ with $v_{i, q}$; then identify $v_{0, j}$ with $v_{k, q-1-j}$ if $k$ is even, and $v_{0, j}$ with $v_{k, q-j}$ if $k$ is odd.

Theorem 13 ([10]). A polyhex graph on the Klein bottle is isomorphic to either $K_{e}(k, q)$ $(q \geqslant 2$, even $k \geqslant 4)$ or $K_{o}(k, q)$ (even $\left.q \geqslant 2, k \geqslant 3\right)$. 

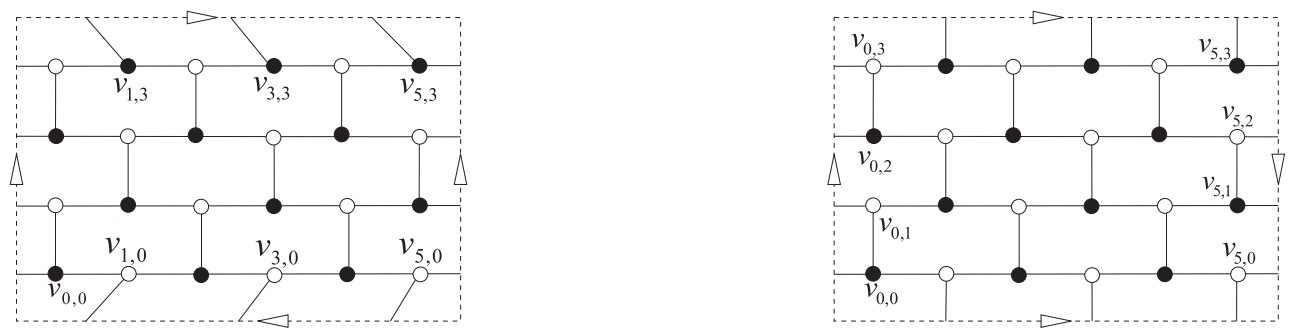

Figure 6: Polyhex graphs $K_{e}(6,4)$ (left) and $K_{o}(6,4)$ (right) on the Klein bottle.

Polyhex graphs contain many interesting graphs. For example, $T(14,1,2)$ is isomorphic to the Heawood graph, both $T(8,1,1)$ and $K_{e}(4,2)$ are isomorphic to the cube, and $T(6,1,1)$ is isomorphic to $K_{3,3}$.

Proposition 14 ([30]). There is a hexagon-preserving isomorphism between $T(k, q, t)$ and $T\left(k, q, t^{\prime}\right)$ where $t^{\prime} \equiv(k-2 q-2 t) / 2(\bmod k / 2)$.

Proposition 15 ([23]). Every polyhex graph on the torus is vertex-transitive and hexagontransitive.

Theorem 16 ([29]). Every bipartite polyhex graph is a brace.

By Theorems 9 and 16, we have the following result.

Corollary 17. Every bipartite polyhex graph is cyclically 4-edge-connected.

Lemma 18. Let $G$ be a polyhex graph on the torus. Then $\mathrm{fw}(G)=2$ if and only if $G$ is isomorphic to $T(k, q, t)$ where integers $k, q, t$ satisfy $(k, q, t) \in\{(4, q, t) \mid q \geqslant 2\}$ or $(k, q, t) \in$ $\{(k, 2, t) \mid k \geqslant 6, t \in\{k / 2-2, k / 2-1,0\}\}$ or $(k, q, t) \in\{(k, 1, t) \mid k \geqslant 6, k / 4-1 \leqslant t \leqslant k / 4\}$.

Proof. Let $G$ be a polyhex graph on the torus. Then $G$ is isomorphic to some $T(k, q, t)$ by Theorem 12. It follows that $\mathrm{fw}(G)=2$ if and only if $G$ has two distinct hexagons $h_{1}$ and $h_{2}$ which intersect at two edges. By Proposition 15, without loss of generality, let $h_{1}$ be the hexagon $h_{0,0}$ and let $h_{2}=h_{x, y}$ with $0 \leqslant y \leqslant q-1$. Since $h_{1}$ and $h_{2}$ intersect at two edges, $y=0$ or 1 .

If $y=1$, then $q=2$. It follows that $x=1$ or $k-1$ since $k>4$. Hence $t \in$ $\{k / 2-2, k / 2-1,0\}$. On the other hand, a polyhex $T(k, 2, t)$ with $t \in\{k / 2-2, k / 2-1,0\}$ does have face-width 2 .

If $y=0$, then either $k=4$ or $q=1$. If $k=4$, then $q \geqslant 2$ by Theorem 12 , and further $\mathrm{fw}(T(4, q, t))=2$. The lemma holds. So suppose $q=1$. Then $v_{i, 0}=v_{i+1+2 t, 1}$ for each $i$. As $h_{1}$ and $h_{2}$ intersect at two edges, $\left\{v_{0,0}, v_{2,0}\right\} \cap V\left(h_{x, 0}\right) \neq \emptyset$ and $\left\{v_{2 x, 0}, v_{2 x+2,0}\right\} \cap V\left(h_{0,0}\right) \neq \emptyset$. Note that $\left\{v_{0,0}, v_{2,0}\right\} \cap V\left(h_{x, 0}\right) \neq \emptyset$ implies

$$
2 x-1 \leqslant 1+2 t \leqslant 2 x+1,
$$


and $\left\{v_{2 x, 0}, v_{2 x+2,0}\right\} \cap V\left(h_{0,0}\right) \neq \emptyset$ implies

$$
k-1 \leqslant 2 x+1+2 t \leqslant k+1 .
$$

Combining inequalities (1) and (2), we have $k / 4-1 \leqslant t \leqslant k / 4$. On the other hand, if $k / 4-1 \leqslant t \leqslant k / 4$ and $k \geqslant 6$, then $h_{0,0}$ and $h_{\lfloor k / 4\rfloor, 0}$ intersect at two edges. So $T(k, 1, t)$ with $k / 4-1 \leqslant t \leqslant k / 4$ and $k \geqslant 6$ has face-width 2 .

By Lemmas 3 and 18, the Heawood graph has an embedding $T(14,1,2)$ on the torus with face-width 3 .

Theorem 19. Let $G$ be a polyhex graph on the Klein bottle. Then $\mathrm{fw}(G)=\min \{\lceil k / 2\rceil, q\}$.

Proof. Let $G$ be a polyhex graph on the Klein bottle. By Theorem 13, $G$ is isomorphic to either $K_{e}(k, q)$ or $K_{o}(k, q)$.

Let $\mathcal{H}$ be a set of hexgons of $G$ such that $\bigcup_{h_{x, y} \in \mathcal{H}} h_{x, y}$ contains a non-contractible curve $\ell$ and $\mathcal{H}$ is minimum. Then $\operatorname{fw}(G)=|\mathcal{H}|$.

First, assume that $G$ is isomorphic to $K_{e}(k, q)$ ( $k$ is even). Let $H_{i}$ be the graph consisting of all hexagons $h_{x, y}$ with $y=i$. If $\mathcal{H} \cap H_{i} \neq \emptyset$ for all $i \in \mathbb{Z}_{q}$, then $|\mathcal{H}| \geqslant q$. So suppose that $\mathcal{H} \cap H_{t}=\emptyset$ for some $t \in \mathbb{Z}_{q}$. Let $E_{t}=\left\{v_{i, t} v_{i, t+1} \mid i+t \equiv 0(\bmod 2), i \in \mathbb{Z}_{k}\right\}$. All hexagons of $K_{e}(k, q)-E_{t}$ induce a tube. Let $R_{j, t}$ be the graph consisting of all hexagons $h_{m, y}$ with $y \in \mathbb{Z}_{q}$, where

$$
m= \begin{cases}j & \text { if } 0 \leqslant y \leqslant t \\ k / 2-j & \text { if } t+1 \leqslant y \leqslant q-1\end{cases}
$$

Denote $E^{j}=\left\{e \mid e=h_{m, y} \cap h_{m, y+1}\right.$ and $\left.y, y+1 \in \mathbb{Z}_{q} \backslash\{t\}\right\} \cup\left\{e_{1}, e_{2}\right\}$ where $e_{1}=h_{j, t-1} \cap h_{j, t}$ and $e_{2}=h_{k / 2-j, t} \cap h_{k / 2-j, t+1}$. Note that $E^{j} \subset E\left(R_{j}\right)$. Then the union of all hexagons of $K_{e}(k, q)-E_{t}-E^{j}$ does not contain a non-contractible closed curve. So $\mathcal{H} \cap R_{j, t} \neq \emptyset$ for any $j \in \mathbb{Z}_{k / 2}$. Hence $|\mathcal{H}| \geqslant k / 2$. It follows that $\mathrm{fw}(G)=|\mathcal{H}| \geqslant \min \{q, k / 2\}$.

On the other hand, each $H_{i}$ and $R_{\lceil k / 4\rceil, t}$ of $K_{e}(k, q)$ contains a non-contratible closed curve. Note that $H_{i}$ has $k / 2$ hexagons and $R_{\lceil k / 4\rceil, t}$ has $q$ hexagons. It follows that $\mathrm{fw}(G) \leqslant \min \{q, k / 2\}$. Hence $\mathrm{fw}(G)=\min \{q, k / 2\}$.

In the following, assume that $G$ is isomorphic to $K_{o}(k, q)$ ( $q$ is even). Let $R_{i}$ be the graph consisting of all hexagons $h_{x, y}$ with $x=i$ and $y \in \mathbb{Z}_{q}$. If $\mathcal{H} \cap R_{i} \neq \emptyset$ for all integer $i \in[0,\lceil k / 2\rceil]$, then $|\mathcal{H}| \geqslant\lceil k / 2\rceil$. So suppose that $\mathcal{H} \cap R_{t}=\emptyset$ for some $t \in \mathbb{Z}_{\lceil k / 2\rceil}$. Denote $E^{t}=\left\{v_{2 t, j} v_{2 t+1, j} \mid j \in \mathbb{Z}_{q}\right\}$. All hexagons of $K_{o}(k, q)-E^{t}$ induce a tube. Let $H_{j}$ be the graph consisting of all hexagons $h_{x, m}$ with $x \in \mathbb{Z}_{\lceil k / 2\rceil \backslash\{t\}}$, where

$$
m= \begin{cases}j & \text { if } 0 \leqslant x \leqslant t \\ q-1-j & \text { if } t+1 \leqslant x \leqslant k / 2-1 \text { and } k \text { is even; } \\ q-j & \text { if } t+1 \leqslant x \leqslant\lceil k / 2\rceil-1 \text { and } k \text { is odd }\end{cases}
$$

Let $E_{j}=\left\{v_{i, m} v_{i, m+1} \mid i+m \equiv 0(\bmod 2), i \in \mathbb{Z}_{k}\right\}$ where $m$ is defined as above. Then the union of all hexagons of $K_{o}(k, q)-E^{t}-E_{j}$ does not contain a non-contractible closed 
curve. Note that the edges in $E_{j}$ are contained by only hexagons from $H_{j}$. So $\mathcal{H} \cap H_{j} \neq \emptyset$ for any $j \in \mathbb{Z}_{q}$. Hence $|\mathcal{H}| \geqslant q$. It follows that $\operatorname{fw}(G)=|\mathcal{H}| \geqslant \min \{q,\lceil k / 2\rceil\}$.

On the other hand, both $H_{q / 2}$ and $R_{i}$ of $K_{o}(k, q)$ contain a non-contractible closed curve. Note that $H_{q / 2}$ has $\left\lceil\frac{k}{2}\right\rceil$ hexagons, and $R_{\lceil k / 2\rceil-1}$ contains $q$ hexagons. So $\operatorname{fw}(G) \leqslant$ $\min \left\{\left\lceil\frac{k}{2}\right\rceil, q\right\}$. This completes the proof.

Lemma 20. Let $G$ be a polyhex graph on the Klein bottle. Then $\mathrm{fw}(G)=2$ if and only if $G$ is isomorphic to either $K_{e}(k, q)$ with $k=4$ or $q=2$, or $K_{o}(k, q)$ with $3 \leqslant k \leqslant 4$ or $q=2$.

Proof. By Theorem 19, $\mathrm{fw}(G)=2$ if and only if $\min \left\{\left\lceil\frac{k}{2}\right\rceil, q\right\}=2$. It follows that $3 \leqslant k \leqslant 4$ or $q=2$.

By Theorem 13, $G$ is isomorphic to either $K_{e}(k, q)$ with $k=4$ or $q=2$, or $K_{o}(k, q)$ with $3 \leqslant k \leqslant 4$ or $q=2$.

\section{$5 \quad$ Pfaffian polyhex graphs}

In this section, we characterize Pfaffian polyhex graphs.

\subsection{Polyhex graphs on the torus}

Let $G$ be a polyhex graph on the torus. If $G$ is planar, then it is Pfaffian. Now assume that $G$ is neither planar nor the Heawood graph. Proposition 11 implies that $G$ must contain an ideal tri-cut if it is Pfaffian.

Lemma 21. Let $G$ be a polyhex graph on the torus. Then $G$ does not contain an ideal tri-cut.

Proof. Let $G$ be a polyhex graph on the torus. By Theorem 12, $G$ can be represented as $T(k, q, t)$ for some suitable triple of integers $(k, q, t)$. Suppose on the contrary that $G$ has an ideal tri-cut $W$. Then $|W|=4$ and $G-W$ has exactly three components, denoted by $G_{1}, G_{2}$ and $G_{3}$, each of which has at least four vertices.

First suppose $q \geqslant 2$. If $T(k, q, t)$ has a layer $L_{i}$ containing at least three vertices of $W$. Then $k \geqslant 6$ as $W$ is independent in $G$. Since $q \geqslant 2$, any other layer $L_{j}(j \neq i)$ contains at most one vertex in $W$. So all $L_{j}-W$ with $j \in \mathbb{Z}_{q} \backslash\{i\}$ are contained in a common component of $G-W$. Note that at least one vertex of every component of $L_{i}-W$ has a neighbor in either $L_{i+1}-W$ or $L_{i-1}-W$. Hence $G-W$ is connected, a contradiction. So every layer $L_{i}$ has at most two vertices in $W$. That implies that $L_{i}-W$ has at most two paths as components. So $G-W$ has at most two components, also a contradiction.

Now suppose $q=1$. By Lemma 15 , let $W=\left\{v_{i_{0}, 0}, v_{i_{1}, 0}, v_{i_{2}, 0}, v_{i_{3}, 0}\right\}$ with $0=i_{0}<i_{1}<$ $i_{2}<i_{3} \leqslant 2 k-2$. Then $L_{0}-W$ has four paths $P_{0}, P_{1}, P_{2}$ and $P_{3}$ such that $v_{i_{\alpha}, 0}$ joins $P_{\alpha}$ and $P_{\alpha+1}$ with $\alpha, \alpha+1 \in \mathbb{Z}_{4}$. Since $G-W$ has three components, precisely two paths from $P_{\alpha}$ 's $\left(\alpha \in \mathbb{Z}_{4}\right)$ belong to a common component of $G-W$. Note that every vertex of $W$ has exactly one neighbor in each component $G_{i}$. So $P_{\alpha}$ and $P_{\alpha+1}$ can not belong 
to the same component of $G-W$. On the other hand, every component has at least 4 vertices. So every $P_{\alpha}$ has at least two vertices. We may assume that $P_{1}$ and $P_{3}$ belong to the same component of $G-W$. Then both $P_{0}$ and $P_{2}$ are components of $G-W$.

Let $v_{m, 0} \in V\left(P_{2}\right)$ be adjacent to $v_{0,0} \in W$. Then $m=k-(1+2 t)$ since $v_{m, 0}=$ $v_{m+1+2 t, 1}=v_{0,1}$. Note that $v_{m+2,0}=v_{k+1-2 t, 0}$ is adjacent to $v_{2,0} \in V\left(P_{1}\right)$. Since $P_{1}$ and $P_{2}$ belong to different components of $G-W, v_{m+2,0} \notin V\left(P_{2}\right)$. Since each $E\left(W, G_{i}\right)$ is a matching and $P_{1}$ and $P_{3}$ belong to the same component of $G-W, v_{m+2,0} \neq v_{i_{2}, 0}$ and thus $v_{m+2,0} \in V\left(P_{3}\right)$. Then $v_{m+1,0}=v_{i_{2}, 0}$, and $v_{m-2,0} \in V\left(P_{2}\right)$ is adjacent to $v_{k-2,0} \in V\left(P_{0}\right)$, which contradicts that $P_{0}$ and $P_{2}$ belong to different components of $G-W$.

By Proposition 11, Theorem 12 and Lemma 21, we immediately have the following result.

Corollary 22. Let $G$ be a polyhex graph on the torus. Then $G$ is Pfaffian if and only if it is planar or isomorphic to the Heawood graph $T(14,1,2)$.

By Theorem 1, planar polyhex graphs embedded on the torus must have face-width two. Using Kuratowski's Theorem that a graph is planar if and only if it contains no $K_{5}$ and $K_{3,3}$ as minor, the following lemma characterizes planar polyhex graphs on the torus.

Lemma 23. A polyhex graph on the torus is planar if and only if it is isomorphic to either $T(4,2, t)$, or $T(8,1, t)$ or $T(k, 2, k / 2-1)$.

Proof. We can see that both $T(4,2, t)$ and $T(8,1, t)$ are isomorphic to the cube $Q_{3}$ and hence are planar. For $T\left(k, 2, k / 2-1\right.$ ), it is isomorphic to $C_{k} \times K_{2}$ (a plane embedding shown in Figure 7).

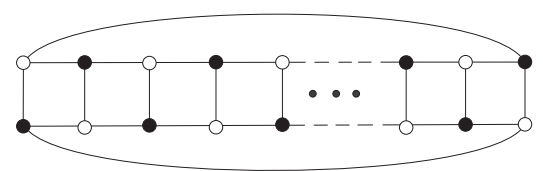

Figure 7: A plane embedding of $T(k, 2, k / 2-1)$.

Next we will show that the other polyhex graphs on the torus are not planar. By Theorem 1 and Lemma 18, it suffices to show that $T(4, q, t)(q \geqslant 3)$, and $T(k, 2, t)(k \geqslant 6$, $t=0$ or $k / 2-2)$, and $T(k, 1, t)(k \geqslant 6, k \neq 8$ and $k / 4-1 \leqslant t \leqslant k / 4)$ are all non-planar.

Note that $T(4, q, t)(q \geqslant 3)$ is non-planar by Kuratowski's Theorem since it contains a subdivision of $K_{3,3}$ as a subgraph (see the subgraphs induced by thick lines in Figure 8).
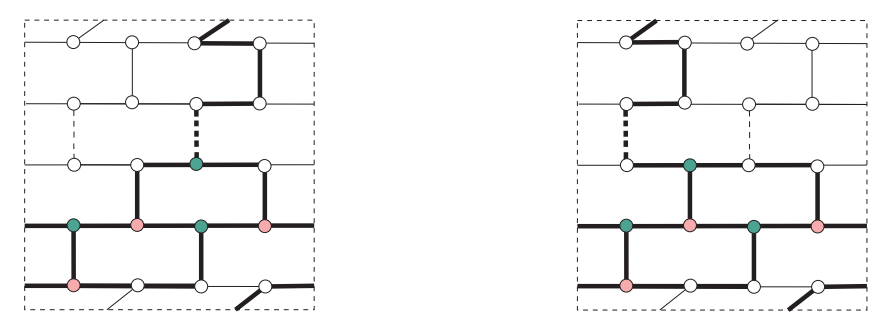

Figure 8: $K_{3,3}$-subdivisions in $T(4, q, t)$ with $q \geqslant 3$. 
By Proposition 14, $T(k, 2,0)$ is isomorphic to $T(k, 2, k / 2-2)$. Since $T(k, 2,0)(k \geqslant 3)$ contains a $K_{3,3}$-minor (see Figure 9 ), both $T(k, 2,0)$ and $T(k, 2, k / 2-2)$ are non-planar.

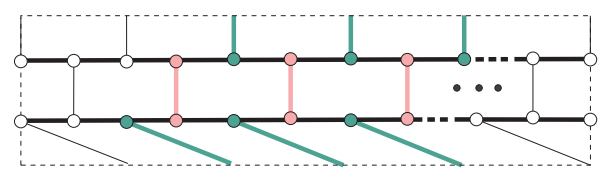

Figure 9: A $K_{3,3}$-minor in $T(k, 2,0)(k \geqslant 6)$.

Now consider $T(k, 1, t)(k \geqslant 6, k \neq 8$ and $k / 4-1 \leqslant t \leqslant k / 4)$. Since $T(6,1,1)$ is isomorphic to $K_{3,3}$, it is thus non-planar. For $T(k, 1, t)$ with $k \geqslant 12$ and $k / 4-1 \leqslant t \leqslant k / 4$, it contains a subdivision of $K_{3,3}$ as a subgraph induced by edges $v_{0,0} v_{k-1-2 t, 0}, v_{2,0} v_{k+1-2 t}$, $v_{k-1,0} v_{v-3-2 t, 0}$ and all edges of $L_{0}$ (see Figure 10 (left)). If $k=10, t=2$. So $T(10,1,2)$ contains a subdivision of $K_{3,3}$ as shown in Figure 10 (right).
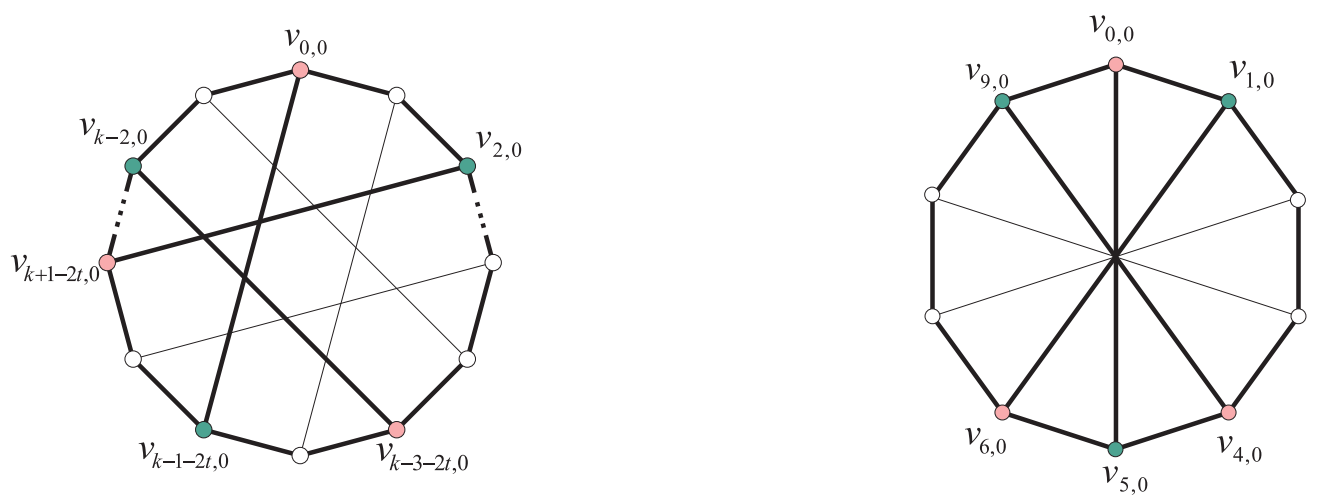

Figure 10: $K_{3,3}$-subdivisions in $T(k, 1, t)(k \geqslant 12$ and $k / 4-1 \leqslant t \leqslant k / 4$ (left)) and $T(10,1,2)$ (right).

By Corollary 22 and Lemma 23, we have the following result which characterizes Pfaffian polyhex graphs on the torus.

Theorem 24. Let $G$ be a polyhex graph on the torus. Then $G$ has a Pfaffian orientation if and only if $G$ is isomorphic to either the Heawood graph, or the cube $Q_{3}$, or $C_{k} \times K_{2}$ for even $k \geqslant 4$.

\subsection{Polyhex graphs on the Klein bottle}

First, we consider bipartite polyhex graphs on the Klein bottle.

Lemma 25. Let $G$ be a bipartite polyhex graph on the Klein bottle. Then $G$ does not contain an ideal tri-cut. 
Proof. By Theorem 13, we have that $G$ is isomorphic to $K_{e}(k, q)$ with $q \geqslant 2$. An analogous argument as the proof of Theorem 21 in case $q \geqslant 2$ shows that the lemma is true.

It can be seen that the Heawood graph can not be a polyhex graph on the Klein bottle. So, by Lemma 25 and Proposition 11, a Pfaffian bipartite polyhex graph on the Klein bottle must be planar.

Lemma 26. A bipartite polyhex graph $G$ on the Klein bottle is planar if and only if it is $K_{e}(4,2)$.

Proof. Let $G$ be a planar bipartite polyhex graph on the Klein bottle. By Theorem 1 and Lemma 20, we may assume that $G$ is isomorphic to $K_{e}(4, q)(q \geqslant 2)$ or $K_{e}(k, 2)(k \geqslant 4)$.

Since $K_{e}(k, 2)(k \geqslant 6)$ contains a subdivision of $K_{3,3}$ (see Figure 11), it is non-planar. Note that $K_{e}(4, q)$ is isomorphic to $T(4, q, 1)$. Since $T(4, q, 1)$ with $q \geqslant 3$ contains a subdivision of $K_{3,3}$, it is non-planar. The polyhex graph $K_{e}(4,2)$ is isomorphic to the cube and hence planar. Hence $G$ is $K_{e}(4,2)$.
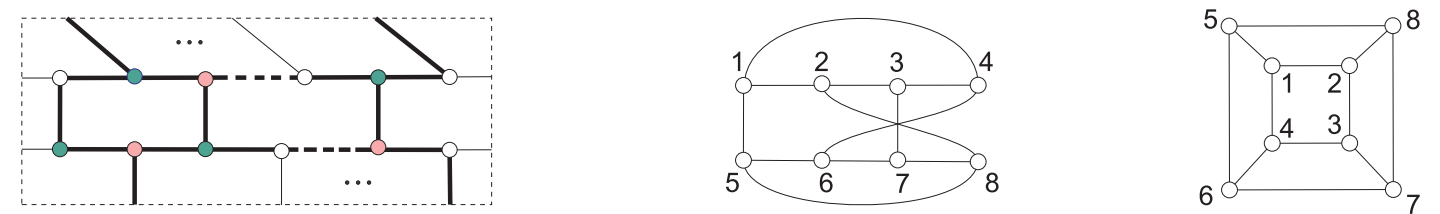

Figure 11: A $K_{3,3}$-subdivision in $K_{e}(k, 2)$ and $K_{e}(4,2)$ is isomorphic to $Q_{3}$.

By Proposition 11, Theorem 8 and Lemmas 25 and 26, we have the following characterization of Pfaffian bipartite polyhex graphs on the Klein bottle.

Theorem 27. Let $G$ be a bipartite polyhex graphs on the Klein bottle. Then $G$ is Pfaffian if and only if it is isomorphic to the cube.

In the following, let $G$ be a non-bipartite polyhex graphs on the Klein bottle. A cycle $C$ of $G$ is 1-sided if its tubular neighborhood is homeomorphic to a Möbius strip, and 2-sided, otherwise. An embedding of a graph $G$ in the Klein bottle is cross-cap-odd if every non-separating cycle $C$ of $G$ has odd size if and only if it is 1-sided.

Lemma 28 ([17]). Every graph that admits a cross-cap-odd embedding in the Klein bottle is Pfaffian.

Lemma 29. The embedding of a non-bipartite polyhex graph $K_{o}(k, q)$ is a cross-cap-odd embedding.

Proof. For $K_{o}(k, q)$, let

$$
E_{0}:= \begin{cases}\left\{v_{0, i} v_{k-1, q-1-i} \mid i \in \mathbb{Z}_{k}\right\}, & \text { if } k \equiv 0(\bmod 2) \\ \left\{v_{0, i} v_{k-1, q-2-i} \mid i \in \mathbb{Z}_{k}\right\}, & \text { otherwise }\end{cases}
$$




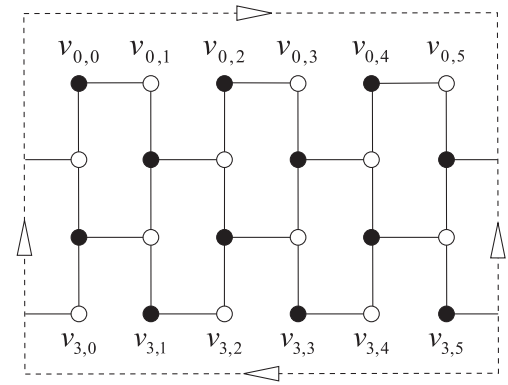

Figure 12: The tube $T_{4,6}$ obtained from $K_{o}(4,6)$ by deleting edges $v_{0, i} v_{3,5-i}$ for $i \in \mathbb{Z}_{6}$.

The subgraph obtained from $K_{o}(k, q)$ by deleting all edges in $E_{0}$, together with its hexagonal faces, is a tube, denoted by $T_{k, q}$. Conversely, $K_{o}(k, q)$ can be generated from the tube $T_{k, q}$ by adding edges in $E_{0}$.

Note that $T_{k, q}$ is a bipartite graph since a proper 2-coloring of $L(k, q)$ is also a proper 2-coloring of $T_{k, q}$. Any edge in $E_{0}$ joins two vertices with the same color. Now for any cycle $C$ of $K_{o}(k, q)$, if $C$ is also a cycle of $T_{k, q}$, then $C$ is 2-sided and has even length. If $C$ is not a cycle of $T_{k, q}$, then $E(C) \cap E_{0} \neq \emptyset$. Let $\delta:=\left|E(C) \cap E_{0}\right|$. Contract every edge in $E_{0} \cap E(C)$ in $C$ to a single vertex, and let $C^{\prime}$ be the new cycle. Then $C^{\prime}$ has even length since the vertices alternate in color along $C^{\prime}$. Hence $|E(C)| \equiv \delta(\bmod 2)$.

Note that the tubular neighborhood of $C$ is homeomorphic to a Möbius strip if and only if $\delta=\left|E(C) \cap E_{0}\right| \equiv 1(\bmod 2)$. So $C$ is 1-sided if and only if $|E(C)| \equiv \delta=\left|E(C) \cap E_{0}\right| \equiv 1$ $(\bmod 2)$. It follows that $K_{o}(k, q)$ is a cross-cap-odd embedding.

By Lemmas 28 and 29, the following result follows immediately.

Theorem 30. Every non-bipartite polyhex graph on the Klein bottle is Pfaffian.

In [10], it has been shown that $K_{o}(k, q)$ is 2-extendable if and only if $k \geqslant 4$ and $q \geqslant 5$. By a result of Lovász and Plummer (Theorem 5.5.23 on Page 206 in [13]) that a 2-extendable graph is either bicritical or elementary bipartitie, $K_{o}(k, q)$ is bicritical and hence a brick. By Theorem 19, the face-width $\mathrm{fw}\left(K_{o}(k, q)\right)=\min \left\{\left\lceil\frac{k}{2}\right\rceil, q\right\} \rightarrow \infty$ as $\min \{k, q\} \rightarrow \infty$. Hence we have the following remark.

Remark 1. Theorem 4 shows that a Pfaffian brace embedded on a surface $\Sigma$ with $g(\Sigma)>0$ has a small face-width. But the face-width of a Pfaffian brick on sufaces with a positive genus could be arbitrarily large.

Remark 2. In [18], Norine and Thomas conjectured that every Pfaffian cubic graph is 3edge colorable. As shown in [28], every polyhex graph on the Klein bottle is Hamiltonian and hence 3-edge colorable. By Theorem 30, every Pfaffian polyhex graph is 3-edge colorable. Hence the conjecture of Norine and Thomas is true for Pfaffian polyhex graphs. 


\section{Acknowledgements}

We would like to thank the anonymous referee for valuable comments to improve the final version.

\section{References}

[1] A. Altschuler. Construction and enumeration of regular maps on the torus. Discrete Math., 4:201-217, 1973.

[2] M. Deza, P.W. Fowler, A. Rassat and K.M. Rogers. Fullerenes as tilings of surfaces. J. Chem. Inf. Comput. Sci., 40:550-558, 2000.

[3] D. Holton and M.D. Plummer. 2-extendability in 3-polytopes. In Combinatorics, Eger, Hungary, 1987, Colloq. Math. Soc. J. Bolyai, Volume 52, pages 281-300. Akadémiai Kiadó, Budapest, 1988.

[4] D. Holton and M.D. Plummer. Matching extension and connectivity in graphs II. In Graph theory, Combinatorics, and Applications, Volume 2, pages 651-665. Wiley, New York, 1991.

[5] P.E. John. Kekulé count in toroidal hexagonal carbon cages. Croat. Chem. Acta, 71(3):435-447, 1998.

[6] P.W. Kasteleyn. The statistics of dimers on a lattice. I. The number of dimer arrangments on a quadratic lattice. Physica, 27:1209-1225, 1961.

[7] P.W. Kasteleyn. Graph theory and crystal physics. In Graph Theory and Theoretical Physics (F. Harary eds.), pages 43-110. Academic Press, NewYork 1967.

[8] E.C. Kirby. Recent work on toroidal and other exotic fullerene structures. In From Chemical Topology to Three-Dimensional Geometry (A.T. Balaban eds.), pages 263296. Plenum Press, New York, 1997.

[9] E.C. Kirby, R.B. Mallion and P. Pollak. Toroidal polyhexes. J. Chem. Soc. Faraday Trans., 89(12):1945-1953, 1993.

[10] Q. Li, S. Liu and H. Zhang. 2-extendability and $k$-resonance of non-bipartite Kleinbottle polyhexes. Discrete Appl. Math., 159(8):800-811, 2011.

[11] C.H.C. Little. A characterization of convertible (0,1)-matrices. J. Combin. Theory Ser. B, 18:187-208, 1975.

[12] L. Lovász. Matching structure and the matching lattice. J. Combin. Theory Ser. B, 43:187-222, 1987.

[13] L. Lovász and M.D. Plummer. Matching Theory. Ann. Discrete Math. 29, NorthHolland, Amsterdam, 1986.

[14] W. McCuaig. Pólya's permanent problem. Electron. J. Combin., 11: \#R79, 83pp, 2004.

[15] B. Mohar and C. Thomassen. Graphs on Surfaces, Johns Hopkins University Press, Baltimore, 2001. 
[16] S. Negami. Uniqueness and faithfulness of embedding of toroidal graphs. Discrete Math., 44:161-180, 1983.

[17] S. Norine. Matching structure and Pfaffian orientations of graphs. PhD Dissertation, Georgia Institute of Technology, 2005.

[18] S. Norine and R. Thomas. Pfaffian labelings and signs of edge-colorings. Combinatorica, 28(1):99-111, 2008.

[19] M.D. Plummer. On n-extendable graphs. Discrete Math., 31:201-210, 1980.

[20] M.D. Plummer. Matching extension in bipartite graphs. Congr. Numer., 54:245-258, 1986.

[21] N. Robertson, P.D. Seymour and R. Thomas. Permanents, Pfaffian orientations, and even directed circuits. Ann. Math., 150:929-975, 1999.

[22] N. Robertson and R.P. Vitray. Representativity of surface embedding. In Paths, Flows, and VLSI-Layout (B. Krote, L. Lovász, H.J. Proömel, and A. Schrijver eds.), pages 293-328. Springer-Verlag, Berlin, 1990.

[23] W.C. Shiu, P.C.B. Lam and H. Zhang. k-resonance in toroidal polyhexes. J. Math. Chem., 38 (4): 451-466, 2005.

[24] R. Thomas. A survey of Pfaffian orientations of graphs. In Proceedings of the International Congress of Mathematicians, Volume 3, pages 963-984. Madrid, 2006.

[25] C. Thomassen. Embeddings of graphs with no short noncontractible cycles. $J$. Combin. Theory Ser. B, 48:155-177, 1990.

[26] C. Thomassen. Tilings of the torus and the Klein bottle and vertex-transitive graphs on a fixed surface. Trans. Amer. Math. Soc., 323:605-635, 1991.

[27] V.V. Vazirani and M. Yannakakis. Pfaffian orientations, 0-1 permanents, and even cycles in directed graphs. Discrete Appl. Math., 25:179-190, 1989.

[28] D. Ye. Hamilton cycles in cubic polyhex graphs on the Klein bottle. Ars Combin., 112:205-212, 2013.

[29] D. Ye and H. Zhang. 2-extendability of toroidal polyhexes and Klein-bottle polyhexes. Discrete Appl. Math., 157:292-299, 2009.

[30] H. Zhang and D. Ye. k-resonant toroidal polyhexes. J. Math. Chem., 44(1):270-285, 2008. 